\title{
Characterization of Solar Panels for Powering Sensor Applications
}

\author{
M. Alves ${ }^{1}$, J.M. Dias Pereira ${ }^{1,2}$, J.P.S. Catalão ${ }^{3,4}$ \\ ${ }^{1}$ ESTSetúbal-LabIM/IPS \\ R. Vale de Chaves, Estefanilha, 2910-761 Setúbal (Portugal) \\ Phone: +351265 790000 \\ e-mail: mario.alves@estsetubal.ips.pt; dias.pereira@estsetubal.ips.pt \\ ${ }^{2}$ Instituto de Telecomunicações, Instituto Superior Técnico \\ Av. Rovisco Pais 1, 1049-001 Lisboa (Portugal) \\ Phone: +351218418 454, Fax: +351218418472 \\ ${ }^{3}$ University of Beira Interior \\ R. Fonte do Lameiro, 6200-001 Covilhã (Portugal) \\ Phone: +351275329 914, Fax: +351275329972 \\ e-mail: catalao@ubi.pt \\ ${ }^{4}$ Center for Innovation in Electrical and Energy Engineering, Instituto Superior Técnico \\ Av. Rovisco Pais 1, 1049-001 Lisbon (Portugal) \\ Phone: +351218417 000, Fax: +351218499242
}

\begin{abstract}
Devices installed in remote places require continuously energy supply for their functionality or communication requirements. For these applications, conventional energy supply is very expensive, or unreliable, and batteries dependence is not a good solution for long-term installations. Energy harvesting can be a good solution, to increase the availability of energy. Regenerative energy sources like thermoelectric, magnetic, piezoelectric, and/or renewable sources such as photovoltaic, wind, among others, allowed the development of different powering solutions for sensor units. The purpose of this work is to characterize three photovoltaic solar panels for different power consumption sensor applications. The study is carried out taking into account the intensity of light and angle of incidence over the photovoltaic panels versus voltage generated. The energy is stored in an association of supercapacitors. This association together with the solar panel itself forms the two possible sources to supply the sensor application.
\end{abstract}

\section{Key words}

Regenerative energy sources, energy harvesting, solar energy, photovoltaic cells, sensor applications.

\section{Introduction}

Nowadays, the increases in efficiency of sources that capture the energy of the surrounding environment, like solar, wind, vibration and temperature gradients, it becomes possible the development of energy autonomous systems or a great increase of autonomy supplied by the batteries.

The problem of autonomous devices self-powered over a full lifetime by harvesting the energy of the environment is the storage of that energy when it isn't completely consumed by the system or when the source can't always delivery the same amount of energy that guarantees the consumption needs.
Solar energy is one of the most used sources of renewable energy. The photovoltaic cells convert to electricity the captured light. Recent developments promise conversion efficiency above $40 \%$ [1] for photovoltaic cells. On the other hand, the use of new and different materials allows reducing drastically the dimension and weight of solar panels for several applications, where small sizes and/or thickness are required.

The goal of this paper is to characterize different size solar panels, in a way of optimizing the efficiency and costs in function of the power systems requirements.

Many energy harvesting systems are oversized, either because there is no controller to manage and optimize the energy flow or because they are designed for worst-case scenarios [2].

The characterization is done by the capability to generate power for instantaneous consumption, or energy storage versus intensity of light and angle of incidence over solar panels.

The experimental results allow determining the point of maximum power generation in each solar panel, thus improving the performances in function of the requirements of each application supply.

For systems installed in the field (open air), the light intensity varies strongly during the day and does not exist during the night. With the daylight, the solar panel must be able to supply sufficient power that allows to support the power requirement of the application and to store the excessive energy. This stored energy is used to support the power of the load, during eventual working hours under less daylight intensity or during the night, when there are no more regenerative/renewable sources able to supply energy to the application. 
The supply requirements for the current devices installed in remote places are characterized for generally low average power and large lifetime energy accumulated. Nowadays, two main storage components can be used: the batteries and the supercapacitors.

The use of supercapacitors has the following advantages: life cycle, with at least two order of magnitude higher than the corresponding one of lead acid batteries; long operation time in a temperature range of $-40^{\circ} \mathrm{C}$ to $+60^{\circ} \mathrm{C}$; higher power capability; able to deliver for short time electrical energy at significantly higher power than batteries [3].

\section{Solar Energy}

Portugal have one of the highest solar irradiation levels within the European Union, around 1400-1800 $\mathrm{kWh} / \mathrm{m}^{2} /$ year (Figure 1). The percentage of sunny days per year is very high, even out of the summer period. For this reason, the implementation and development of systems that use this source of energy in outdoor applications make perfect sense in a large scale, starting in energy/power supply of a small sensor application.

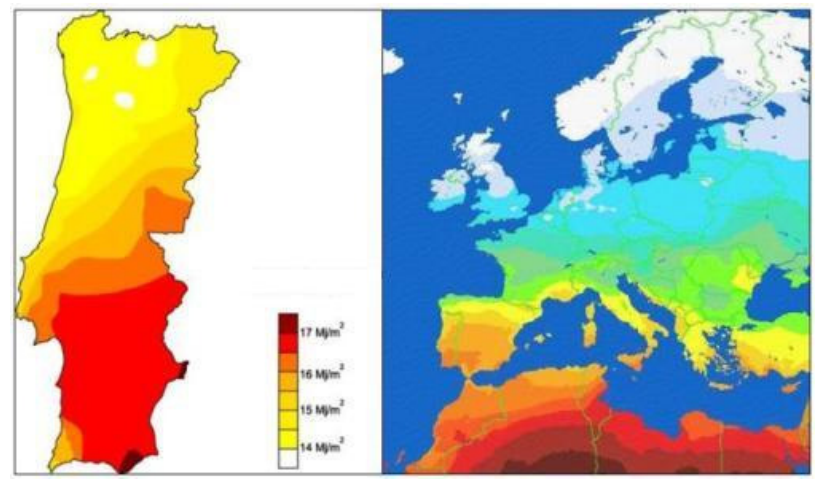

Fig. 1. Daily horizontal solar irradiation in Portugal.

The usage of a solar harvesting system has particular interest for electrical energy generation. Solar cells represent the fundamental power conversion unit of a photovoltaic system [4].

\section{A. Solar Cells}

In this paper we focus our work in three panels of monocrystalline solar cells (Table I) of same technology, but with different dimensions, implying different values of voltage, current, power and, consequently, different power sourcing capabilities.

Table I. Dimension of panels cells.

\begin{tabular}{|c|c|}
\hline Panel & Dimension $(\mathrm{cm})$ \\
\hline $\mathrm{A}$ & $9 \times 6$ \\
\hline $\mathrm{B}$ & $11 \times 11$ \\
\hline $\mathrm{C}$ & $60 \times 26$ \\
\hline
\end{tabular}

The power generation of solar cells is strongly influenced by the illumination condition, such as the intensity and angle of incidence of sunlight along the day. The variation with the intensity will be analyzed in Section 3 .
Regarding the incidence range, it was observed that the position of solar cells is very important to improve the power generation, with more influence relatively to the Y-axis, where the rotation of $45^{\circ}$ in incidence of sunlight has a reduction in short-circuit current of about 25 percent.

Solar Cells exhibit a strong nonlinear electrical characteristic, which makes it low effective extracting energy in non stationary environments [5].

\section{B. Power and Energy Harvesting}

The present work focuses on characterizing solar cells when they are directly connected to a load or to a storage element. For a specific application and environment conditions it is very important to analyze the behavior of the power supply that better fulfills the system requirements. However, harvesting components, such as solar panels, and energy storage elements, such as batteries or ultracapacitors, have different voltage-current characteristics, which must be matched to each other as well as the energy requirements of the system to maximize harvesting efficiency [6].

In $[4,5,7]$ several maximum power point (MPP) tracking techniques and direct methods were developed and compared using experimental results. In [8] it was presented a $\mathrm{DC} / \mathrm{DC}$ boost converter for very low voltage supply $(200 \mathrm{mV})$.

Several other approaches are being investigated to optimize the MPP of other type of power harvesting sources, which can be found in different papers. These developments are very important, since they can improve the efficiency and, above all, enable solutions to a wider range of applications. However, they have power consumption and are no longer a low price solution. The knowledge of all factors and the limitation of the solution for a very specific application can maximize the efficiency and avoid the use of these circuits.

\section{Discussion of Experimental Results}

The open circuit voltage $\left(\mathrm{V}_{\mathrm{OC}}\right)$ and the short circuit current $\left(\mathrm{I}_{\mathrm{SC}}\right)$ are the two main parameters that allow to characterize the solar panels. These parameters permit to analyze each panel by the equivalent Thevenin circuit (Figure 2).

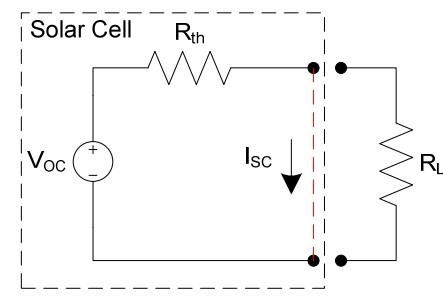

$$
R_{t h}=\frac{V_{O C}}{I_{S C}}
$$

Fig. 2. Equivalent Thevenin Circuit.

Figure 3 represents how $\mathrm{V}_{\mathrm{OC}}$ and $\mathrm{I}_{\mathrm{SC}}$ vary with the solar light during a cloudy day, between 11:00 and the 17:15. 


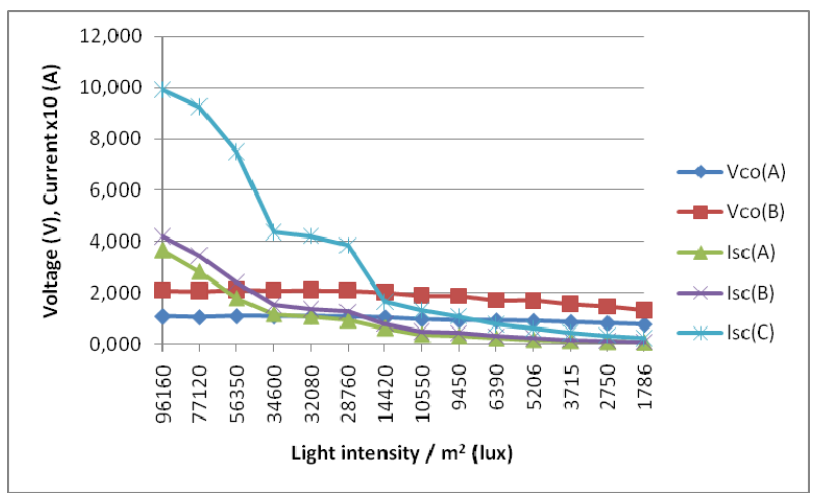

Fig. 3. The three panels $\mathrm{V}_{\mathrm{OC}}$ and $\mathrm{I}_{\mathrm{SC}}$ in function of light intensity.

As can be observed the panel terminals voltage $\left(\mathrm{V}_{\mathrm{OC}}\right)$ is almost constant during the day, in opposite to the current $\left(\mathrm{I}_{\mathrm{SC}}\right)$ that has a strong nonlinear variation during the day.

Consequently, the MPP it is going to move along the day, for a determined load or storage system connected to panel terminals. The maximum power, comparing the panels A and B (more similar) is mostly influenced by the double value of voltages than by the currents, which are almost equal in the middle of the afternoon.

Panel $\mathrm{C}$ has a very similar characteristic, but for higher levels of voltage and current. In other words, the three panels have very similar sensitivity to the intensity of sunlight, in terms of behavior, but with differences in value, which can be achieved for very specific intervals of sunlight. Inside these four intervals we can observe that they have almost a linear characteristic.

Considering the equivalent Thevenin circuit of each solar panel, it is possible to determine the MPP in function of the relation between $\mathrm{V}_{\mathrm{OC}}$ and $\mathrm{I}_{\mathrm{SC}}$.

$$
P=\frac{V_{O C}^{2}}{R_{t h}+R_{L}} \quad P_{\max }=\frac{d P}{d R_{L}} \Rightarrow R_{t h}=R_{L}
$$

For example, for panel $A, R_{\text {th }}$ varies from $2,96 \Omega$ to $117,58 \Omega$, and in panel $\mathrm{B}, \mathrm{R}_{\mathrm{th}}$ varies from $5 \Omega$ and $149,7 \Omega$. This indicates that when connected to a storage system panel A will be faster to obtain one time constant, than panel B. However, its charge level will be lower, limited by the voltage value.

Another 'possible approach is to observe the behavior of the panels directly connected to different loads.

Figures, 4, 5 and 6 represent the response of panels A and $\mathrm{B}$ for two almost fixed values of intensity of sunlight, during 45 minutes of a cloudy afternoon.

The values of current and voltage various drastically around the value of $R_{L}=R_{t h}$, corresponding to the MPP extracted from the panel. The MPP occurs very close to $\mathrm{R}_{\mathrm{L}}$, as expected by the theoretical results. For example, for the lower light intensity it was obtained a value of $19,31 \mathrm{~mW}\left(\mathrm{R}_{\mathrm{L}}=26 \Omega\right)$ in panel $\mathrm{A}$ and a value of $39,34 \mathrm{~mW}\left(\mathrm{R}_{\mathrm{L}}=50 \Omega\right)$ in panel $\mathrm{B}$.

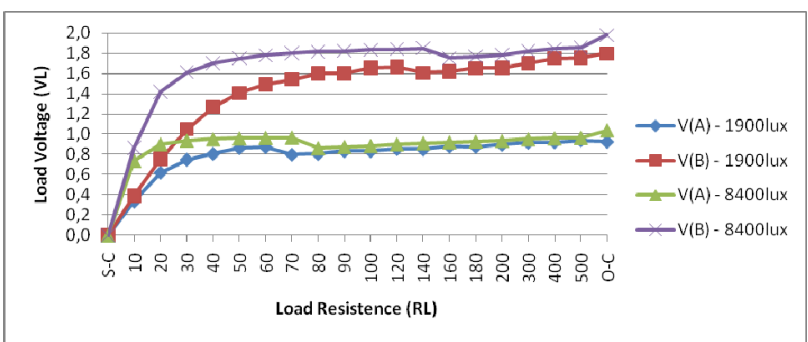

Fig. 4. Panels A and B - Load voltage.

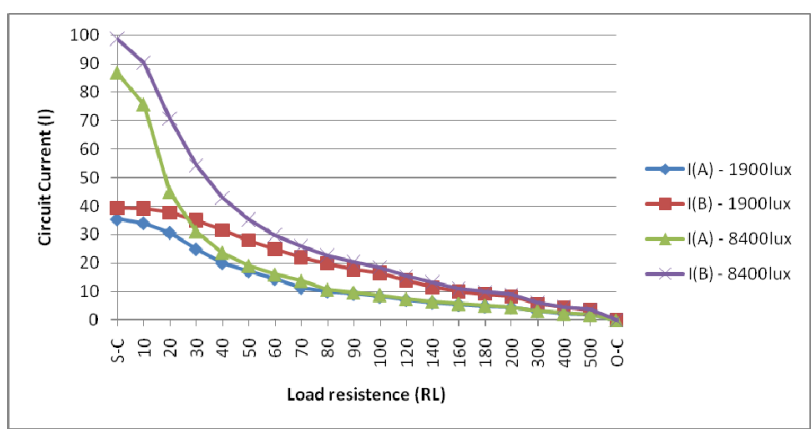

Fig. 5. Panels A and B - Current.

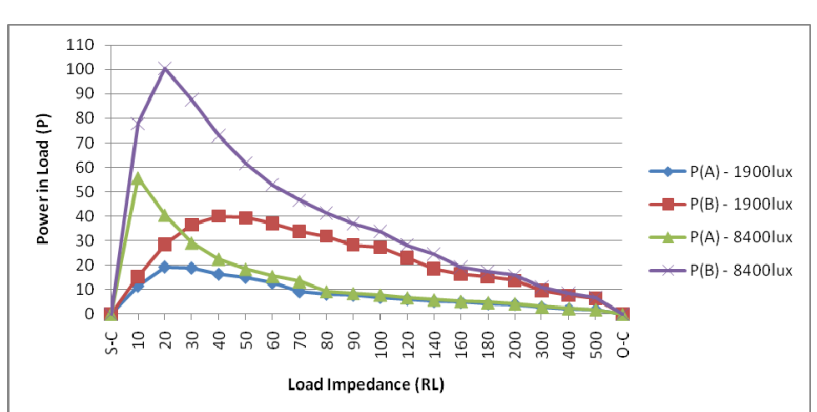

Fig. 6. Panels A and B - Power supplied to the load.

These results, in particularly the one represented in Figure 6, show how important can be the knowledge of the load that is connected to the panel. However, the direct supply of the application from the panels can be difficult, because in the more interesting working range (near MPP) the voltage is not stable and depends on the value of impedance load, as represented in Figure 4.

Two considerations are possible: our application is in a very stable environment (indoor ambient) with a stable level of light intensity, and a constant load impedance value or a storage energy system is required to guarantee a constant value of voltage supply. In practice, the first consideration is less probable, being the second one more usual.

Two solutions are possible: the batteries which have a higher energy density (ideal for long time application voltage supply) and supercapacitors with higher power density to handle short power requirement, as needed in wireless systems to transmit or receive small bit/rates of data (sensors information), periodic in time. For example, in an instrumentation wire fieldbus networks with a conversion wireless module, it is possible to communicate with a wireless node that is monitoring a temperature (with a thermocouple) and is power harvested to supply the wireless system during transmission. 
Panels A and B are useful for systems with low voltage power requirements. They can be associated to increase the voltage value. In this paper, we analyze the isolated behavior of each panel to charge two $2,5 \mathrm{~V}$ supercapacitors, one of $1 \mathrm{~F}$ and the other of $10 \mathrm{~F}$.

The results presented in Figures 7 and 8 show the time required to charge each supercapacitor for a value of light intensity proximally equal to 8400 lux. Table II represents the $\mathrm{V}_{\mathrm{OC}}$ and $\mathrm{I}_{\mathrm{SC}}$ that were obtained for each panel before connection.

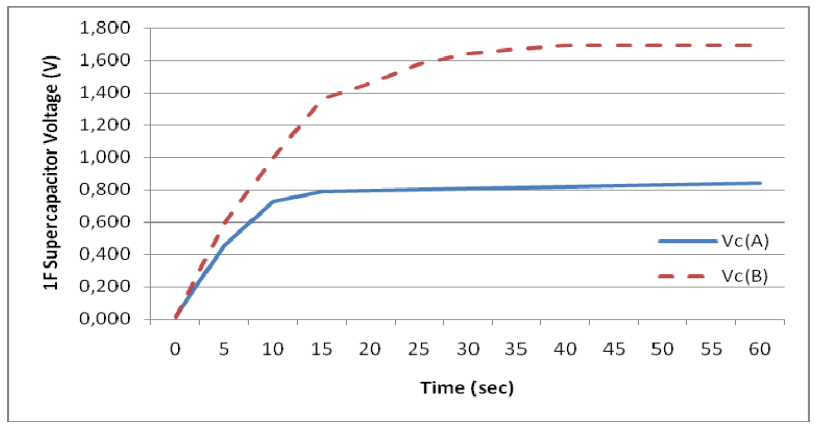

Fig. 7. Supercapacitor (1F) charge with Panels A and B.

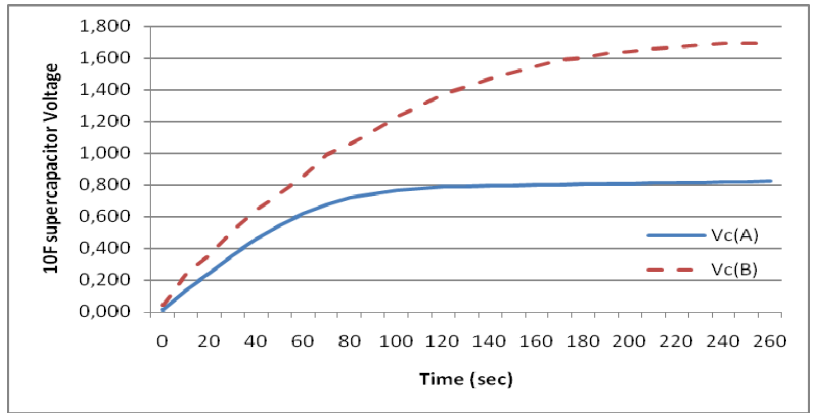

Fig. 8. Supercapacitor (10F) charge with Panels A and B.

Table II. Open circuit voltage and short circuit current.

\begin{tabular}{|c|c|c|}
\hline Panel & $\mathrm{V}_{\mathrm{OC}}(\mathrm{V})$ & $\mathrm{I}_{\mathrm{SC}}(\mathrm{mA})$ \\
\hline A & 0,986 & 86,92 \\
\hline B & 1,957 & 98,77 \\
\hline
\end{tabular}

Each panel was connected to the supercapacitor, through a Schottky diode that is used to prevent reverse current flow into the panel and to maintain a constant voltage across the solar panel terminals (Figure 9).

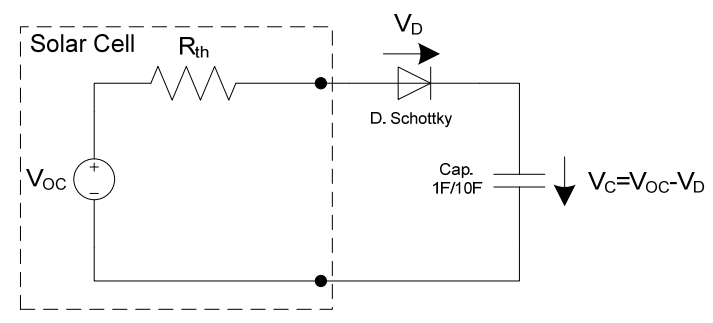

Fig. 9. Supercapacitor connected to the solar cell.

The voltage obtained in each supercapacitor corresponds to the solar cell voltage less the diode voltage drop, of proximally $0,2 \mathrm{~V}(0,32 \mathrm{~V}$ at $1 \mathrm{~A}$ in datasheet). As it can be observed (Table III), panel A is faster than panel B, according to the results represented in Figure 6, since MPP is obtained earlier in panel A.
Table III. One time constant for supercapacitors charge.

\begin{tabular}{|l|c|c|c|c|}
\hline Panel & A (1F) & A (10F) & B (1F) & B (10F) \\
\hline Voltage (V) & 0,548 & 0,548 & 1,087 & 1,087 \\
\hline$\sigma(\mathrm{sec})$ & 7 & 63 & 13 & 84 \\
\hline
\end{tabular}

Panel $\mathrm{C}$ is indicated for higher power applications $(20 \mathrm{~W})$, like a meteorological station where the energy for supplying the several sensors and conditioning signal circuits is more significant than the power requirement for data transmission. For this application a battery of $12 \mathrm{~V} / 18 \mathrm{Ah}$ is more adequate.

\section{Conclusion and Future Work}

Sunlight is a viable choice of increasing the autonomy of outdoor sensorial applications. However, the design of an efficient solar harvesting system involves a full understanding of several factors. Experimental results are shown in this paper considering three power generation levels based on solar power harvesting, for different energy/power requirements of sensors applications. The correct characterization of environmental conditions, load impedance, power/energy supply needs and storage elements are very important to optimize the efficiency of a specific application. Future work will be dedicated to study the behavior of the solar panels as a function of temperature and wave length of light, for different light sources types.

\section{References}

[1] A. B. da Cunha and D. C. Da Silva, "Energy-Efficient Characterization of Solar Panel-Supercapacitors Systems for Energy-Harvesting Aware Wireless Sensor Nodes", in: Proc. 2009 IEEE 20th International Symposium on Personal, Indoor and Mobile Radio Communications, Tokyo, Japan, pp. 2275-2279, 13-16 Sept. 2009.

[2] J. W. Kimball, B. T. Kuhn, and R. S. Balog, "A System Design Approach for Unattended Solar Energy Harvesting Supply", IEEE Transactions on Power Electronics, Vol. 24, No. 3-4, pp. 952-962, Mar.-Apr. 2009.

[3] V. V. N. Obreja, "On the Performance of Commercial Supercapacitors as Storage Devices for Renewable Electrical Energy Sources", in: ICREPQ'07, Sevilla, Spain, Mar. 2007.

[4] C. Hua, C. Shen, "Comparative Study of Peak Power Tracking Techniques for Solar Storage System", in: 13th Annual Power Electronics Conference and Exposition, APEC' 98, Vol. 2, pp. 679-685, Feb. 1998.

[5] C. Alippi and C. Galperti, "An Adaptive System for Optimal Solar Energy Harvesting in Wireless Sensor Network Nodes", IEEE Transactions on Circuit and Systems-I, Vol. 55, No. 6, pp. 1742-1750, 2008.

[6] V. Raghunathan, A. Kansal, J. Hsu, J. Friedman, and M. Srivastava, "Design Considerations for Solar Energy Harvesting Wireless Embedded Systems", in: ISPN 2005, pp. 457-462, April 2005.

[7] D. Brunelli, C. Moser, L. Thiele, and L. Benini, "Design of a Solar-Harvesting Circuit for Batteryless Embedded Systems", IEEE Transactions on Circuit and Systems-I, Vol. 56, No.11, pp. 2519-2528, Nov. 2009.

[8] A. Richelli, L. Colalongo, S. Tonoli, and Z. Kovács, "A $0.2 \mathrm{~V}-1.2 \mathrm{~V}$ Converter for Power Harvesting Applications", in: ESSCIRC 2008, Edinburg, pp. 406-409, 2008. 